\title{
Efficacy of intraoperatively prepared cell- based constructs for bone regeneration
}

\author{
Yang Zhang ${ }^{1}$, Eline C Grosfeld', Winston A Camargo ${ }^{1}$, Hongbo Tang ${ }^{1,2}$, Angela M P Magri ${ }^{1,3}$ \\ and Jeroen J J P van den Beucken ${ }^{1 *}$ (D)
}

\begin{abstract}
Background: Conventional cell-based bone regeneration suffers from the major disadvantage of limited cell supply, time-consuming in vitro expansion cultures, and limited patient-friendliness related to cell isolation and multiple visits to the clinic. Here, we utilized an alternative concept using "easy access cells" that can be obtained in an intraoperative manner to prepare cell-based constructs.
\end{abstract}

Methods: We used stromal vascular fraction (SVF) from human adipose tissue and human monocytes for intraoperative preparation of bone constructs. Conventional constructs grafted with expanded human adipose tissue mesenchymal stem cells (ADMSCs) derived from the same donor were set as positive controls. Additionally, we combined both cell types either or not with monocytes. The cellular interaction of human SVF and ADMSCs with human monocytes was evaluated in vitro. The feasibility and bone-regenerative capacity of intraoperative constructs were determined histologically and histomorphometrically in a rat femoral condyle bone defect model.

Results: SVF displayed equal in vitro osteogenic differentiation compared to donor-matched expanded ADMSCs, which for both was significantly enhanced upon co-culture with monocytes. Moreover, SVF and ADMSCs displayed different immunoregulatory effects on monocytes/macrophages. Upon implantation in rat femoral bone defects, SVF constructs demonstrated superior bone formation compared to ADMSC constructs and cell-free controls; no effects of monocyte addition were observed.

Conclusion: In conclusion, we here demonstrate the feasibility of intraoperative SVF construct preparation and superior bone-regenerative capacity thereof compared to donor-matched ADMSC constructs. The superiority of SVF constructs was found to be linked to the distinct differences between immunoregulatory effects of SVF and ADMSCs.

Keywords: Easy access cells, Intraoperative preparation, Stromal vascular fraction, Adipose-derived cells, Macrophages, Bone regeneration

\section{Background}

Bone tissue engineering concepts have been proposed and conducted over the last decades to meet the increasing need for bone-regenerative therapies in clinics. Their development aims to overcome the limitations of allografts and autografts, which are restricted by limited supply, immunological rejection, and disease transmission issues $[1$, 2]. In bone tissue engineering-based constructs, mesenchymal stromal cells (MSCs) [3] have been mostly used as an autologous cell source to boost the bone regeneration

\footnotetext{
* Correspondence: Jeroen.vandenbeucken@radboudumc.nl 'Department of Biomaterials, PO Box 9101, 6500HB Radboudumc, Nijmegen, the Netherlands

Full list of author information is available at the end of the article
}

process. Generally, these procedures involved bone marrow isolation, MSC expansion in vitro, MSC seeding on a scaffold material and priming osteogenic differentiation in vitro, and finally construct implantation into the bone defect of the patient [4]. This conventional approach has several drawbacks regarding tissue harvest via bone marrow aspiration and time-consuming expansion procedure due to low yield of MSCs in the bone marrow [5]. An alternative in the form of liposuction has become available after the first reports on the existence of high yield of MSCs within adipose tissue with osteogenic differentiation capacities [6, 7].

Although recent work has clearly demonstrated the feasibility and efficacy of using human adipose tissue 
MSCs (ADMSCs) to generate bone constructs in pre-clinical animal studies [8,9], clinical application still is limited due to the disadvantages of the preparation process. To generate ADMSCs, the harvested heterogeneous cell population requires purification and expansion in vitro. In addition, inconvenience from two surgical procedures, for respectively cell harvest and construct implantation, is an obstacle. In order to attain simplicity, practicality, and cost-efficiency, the manufacturing process of bone constructs should ideally be compatible with the timeline of a single surgical procedure. In this case, tissue harvest, cell isolation, cell seeding onto a scaffold and subsequent implantation of a construct should occur within a few hours in an intra-operative manner, without ex vivo cell culture. In this perspective, stromal vascular fraction (SVF), the primary isolated cell population from a lipoaspirate, has more potential over ADMSCs.

SVF is a heterogeneous mixture of stromal cells, endothelial cells, pericytes, lymphocytes, mast cells, and pre-adipocytes [10-12]. Recent work has already demonstrated the feasibility of using this SVF for a one-step surgical approach to generate bone constructs $[13,14]$. From preparation to implantation, this process can be finalized in few hours (less than $4 \mathrm{~h}$ ) and has shown superior bone formation compared to cell-free scaffolds. To date, however, no scientific data report on the performance of SVF constructs compared to MSC-constructs, specifically for both types of constructs containing cells of the same human donor. Additionally, previous studies have shown beneficial effects of cell-cell interactions by applying multiple cell types in cell-based constructs $[15,16]$. Other cells that can be easily isolated are monocytes (MO) from blood. Interestingly, monocytes have shown to stimulate osteogenic events by attracting MSCs and stimulating MSCs to proliferate and/or osteogenically differentiate $[17,18]$. This observation justifies further translational research on the applicability of co-culture strategies using these two types of "easy access cells," i.e., SVF and monocytes, to intraoperatively prepare bone constructs with superior osteogenic potential.

We here aimed to evaluate cellular interaction of human SVF and ADMSCs with human monocytes in vitro and their effects on bone regeneration in vivo. More importantly, we comparatively evaluate the bone regenerative efficiency of tissue-engineered constructs in an intraoperative manner (i.e., without in vitro expansion culture) and those in the conventional manner (i.e., with in vitro expansion culture).

\section{Methods}

\section{Human cell isolation and culture}

Human subcutaneous adipose tissue from three healthy donors (donor 1, female, 42 years old; donor 2, male, 38 years old; donor 3, female, 52 years old) was obtained from the
Department of Plastic Surgery (Radboudumc) after ethical approval (Commissie Mensgebonden Onderzoek; dossier number \#3252) and informed consent. The adipose tissue was excised into pieces and then minced in a $0.1 \%$ collagenase type II (Mannheim, Germany) solution with 1\% bovine serum albumin (BSA, Sigma, St. Louis, USA) for $1 \mathrm{~h}$ at $37{ }^{\circ} \mathrm{C}$ under shaking conditions, as described previously [19]. After centrifugation at $600 \mathrm{~g}$ for $10 \mathrm{~min}$ at room temperature, the supernatant together with the fat layer was discarded. The resulting cell pellet was washed twice with phosphate-buffered solution (PBS; Gibco, Merelbeke, Belgium) with subsequent filtration through a polypropylene membrane with a pore size of $100 \mu \mathrm{m}$. After centrifugation, the cell pellet was suspended in proliferation medium, consisting of $\alpha$-MEM (Gibco, Merelbeke, Belgium) containing 10\% fetal bovine serum (FBS; Gibco, Carlsbad, USA). Red blood cells were lysed by incubation for $2 \mathrm{~min}$ in a solution of $7.49 \mathrm{~g} / \mathrm{L} \mathrm{NH}_{4} \mathrm{Cl}(2.06 \mathrm{~g} / \mathrm{L}$ Tris Base, $\mathrm{pH}$ 7.2). Following centrifugation, the deposited cell aggregates were denoted as SVF, suspended in proliferation medium, and counted using a hemocytometer. This SVF was either frozen as $3 \times 10^{6}$ cells per tube in the liquid nitrogen or used for further culturing to obtain a homogeneous population of ADMSCs.

For culturing, SVF containing $3 \times 10^{6}$ cells was plated in $75-\mathrm{cm}^{2}$ tissue culture flasks (Greiner Bio-one, Frickenhausen, Germany) in proliferation medium supplemented with $1 \mathrm{ng} / \mathrm{ml}$ of basic fibroblast growth factor (bFGF, Sigma-Aldrich, St. Louis, USA). Cells were grown in a humidified incubator at $37{ }^{\circ} \mathrm{C}$ in an atmosphere of $5 \% \mathrm{CO}_{2}$. Two days after seeding, the adherent cells were thoroughly rinsed with PBS to remove non-adherent cells from the culture. In this way, a homogeneous population of ADMSCs was obtained. When reaching around 80\% confluency, cells were detached with $0.05 \%$ trypsin in 0.5 mM EDTA (Sigma-Aldrich, St. Louis, USA) and were passaged. SVF and ADMSCs were characterized for surface marker expression by flow cytometry for the mesenchymal markers CD73 (APC-conjugated, BD Bioscience), CD90 (PE-Cy5-conjugated, BD Bioscience), CD105 (PE-conjugated, BD Bioscience, Franklin Lakes, USA), and negatively against CD45 (PE-conjugated, BD Bioscience). Osteogenic differentiation capacity of ADMSCs was confirmed by 4-week cultures in osteogenic medium (proliferation medium supplemented with $10 \mathrm{nM}$ dexamethasone, $100 \mu \mathrm{M}$ ascorbic acid, and $10 \mathrm{mM} \beta$-glycerophosphate).

The human monocytic cell line THP-1 was purchased from the American Type Culture Collection (ATCC; Manassas, USA) and cultured in RPMI-1640 medium (Gibco, Carlsbad, USA) supplemented with 10\% FBS.

\section{Scaffold material for construct preparation}

Tricalcium phosphate (TCP) granules were kindly provided by Cam Bioceramics (Leiden, the Netherlands). 
Pore sizes of TCP granules varied from 500 to $2 \mathrm{~mm}$, and the granules had an overall porosity of $84 \%$. After sterilization by gamma irradiation (SynergyHealth, Ede, the Netherlands), $11 \mathrm{mg}$ (total volume around $21 \mathrm{~mm}^{3}$ corresponding to the bone defect size for in vivo experimental work) of these granules per sample were placed into individual 2-ml sterile Eppendorf tubes.

\section{Procedure for construct preparation}

Before construct preparation, $3 \times 10^{6}$ SVF cells (cryo-preserved) were thawed, counted, and suspended in $300-\mu$ proliferation medium to be loaded dropwise on top of TCP granules, which were pre-incubated in FBS for $24 \mathrm{~h}$ (hereafter referred to as "SVF groups"). The effective cryopreservation and retention of viability and differentiation capacity of SVF cells was previously shown [20]. The purified ADMSCs from SVF were expanded in proliferation medium with bFGF as described above. Osteogenic medium was applied for ADMSCs 3 days before preparation. $1 \times 10^{6}$ ADMSCs were counted by a hemocytometer and suspended in 300- $\mu$ l proliferation medium and were then loaded onto the granules (hereafter referred to as "ADMSCs groups"). All tubes were then supplemented with $300-\mu \mathrm{l}$ proliferation medium and incubated at $37{ }^{\circ} \mathrm{C}$ for $2 \mathrm{~h}$. Non-attached cells were collected and centrifuged before resuspension in the same medium and similarly dropwise loaded onto granules again. The process was repeated twice during this 2-h period. After that, cells with granules were collected for analysis or transferred into 24-well cell culture plates. Cell attachment was confirmed through 4',6-diamidino-2-phenylindole (DAPI) staining and was quantified by DNA content quantification to ascertain comparable numbers of attached cells. After 2-h incubation, $1 \times 10^{6}$ monocytes suspended in $200-\mu$ proliferation medium were added to the SVF or ADMSCs constructs to make the SVF+MO and ADMSCs+MO groups. Same constructs were prepared for the animal study by directly adding monocytes to the defect sites during the surgery. TCP granules supplemented with the same volume of proliferation medium or $1 \times 10^{6}$ monocytes only were set as the control and MO groups, respectively (Fig. 1a).

\section{In vitro assessment}

\section{Cell adhesion and viability on constructs}

Cell attachment and cellular morphology on constructs were checked before implantation by identifying nuclei and actin of attached cells. The scaffolds were incubated in $20 \mu \mathrm{M}$ Alexa Fluor 568 Phalloidin (Thermofisher Scientific, Breda, Netherlands) for $15 \mathrm{~min}$ and in $300 \mathrm{nM}$ DAPI stain for $5 \mathrm{~min}$ and then quantified with a Zeiss AxioPlan immunofluorescence microscope (Carl Zeiss Microimaging $\mathrm{GmbH}$, Gottingen, Germany). Additionally, cell viability was assessed using a LIVE/DEAD ${ }^{\circledR}$ viability/ cytotoxicity kit for mammalian cells (Invitrogen, Gaithersburg, MD, USA) according to the manufacturer's instructions.

\section{Cell proliferation and osteogenic differentiation on constructs}

Constructs from the above six groups were cultured in osteogenic medium up to 4 weeks. After washing with PBS once, constructs were placed in $1 \mathrm{ml}$ MilliQ and subjected to 2 cycles of freezing and thawing. Cell proliferation was evaluated by assessing the DNA content using a DNA quantification kit (Invitrogen, Carlsbad, $\mathrm{CA}$ ) according to the manufacturer's instructions. Osteogenic differentiation of all constructs was analyzed by quantification of alkaline phosphatase (ALP) activity of cell lysates using a 4-nitrophenyl phosphate-based method [19] after 1, 2, and 4 weeks of culture. To test mineralization, same type and number of cells without TCP granules were seeded in 24-wells cell culture plates. After 4 weeks, mineralization was evaluated by deposit calcium as described earlier [19]. Same cells cultured in the proliferation medium without osteogenic supplements were set in parallel as non-differentiation controls.

\section{Effect of SVF or ADMSCs on macrophages polarization}

To assess the effect of SVF or ADMSCs on macrophages polarization, $1 \times 10^{6}$ ADMSCs or $3 \times 10^{6}$ SVF were seeded on 11-mg TCP granules for $2 \mathrm{~h}$ as described above. $1 \times 10^{6}$ THP-1 monocytes were seeded with $250 \mathrm{nM}$ phorbol myristate acetate (PMA; monocytes were activated into macrophages) for $48 \mathrm{~h}$ in 24-well plates. These cell-loaded constructs and TCP granules were then transferred into 0.4-mm-pore size Corning transwell inserts (Sigma-Aldrich, Zwijndrecht, the Netherlands) and placed into the plates. Co-cultures were incubated for another $48 \mathrm{~h}$. Wells supplemented with $100 \mathrm{ng} / \mathrm{ml}$ lipopolysachariden (LPS; Sigma-Aldrich, St Louis, MO, USA) and $20 \mathrm{ng} / \mathrm{ml}$ interferon- $\gamma$ (IFN- $\gamma$; Sigma-Aldrich, St Louis, MO, USA) or $20 \mathrm{ng} / \mathrm{ml}$ interlukine-4 (IL-4; Sigma-Aldrich, St Louis, MO, USA) and $20 \mathrm{ng} / \mathrm{ml}$ interlukine-13 (IL-13; Sigma-Aldrich, St Louis, MO, USA) were set as control. The secreted tumor necrosis factor $\alpha$ (TNF- $\alpha)$ and transforming growth factor $\beta$ (TGF- $\beta$ ) in the supernatants were evaluated using ELISA kits (e-Bioscience, San Diego, CA, USA) according to the manufacturer's instructions.

\section{Animal surgery}

The study was approved by the Centrale Commissie Dierproeven (CCD; project 2015-137); national guidelines for animal care and welfare were obeyed, conforming to the ARRIVE guidelines. A total of 46 nude rats 


\begin{tabular}{ll}
\hline Aroup name & Cells \\
\hline Control & No cells \\
MO & $1 \times 10^{6}$ monocytes \\
SVFs & $3 \times 10^{6} \mathrm{SVF}$ \\
SVFs + MO & $3 \times 10^{6}$ SVF and $1 \times 10^{6}$ monocytes \\
ADMSCs & $1 \times 10^{6}$ ADMSCs \\
ADMSCs + MO & $1 \times 10^{6}$ ADMSCs and $1 \times 10^{6}$ monocytes
\end{tabular}

B
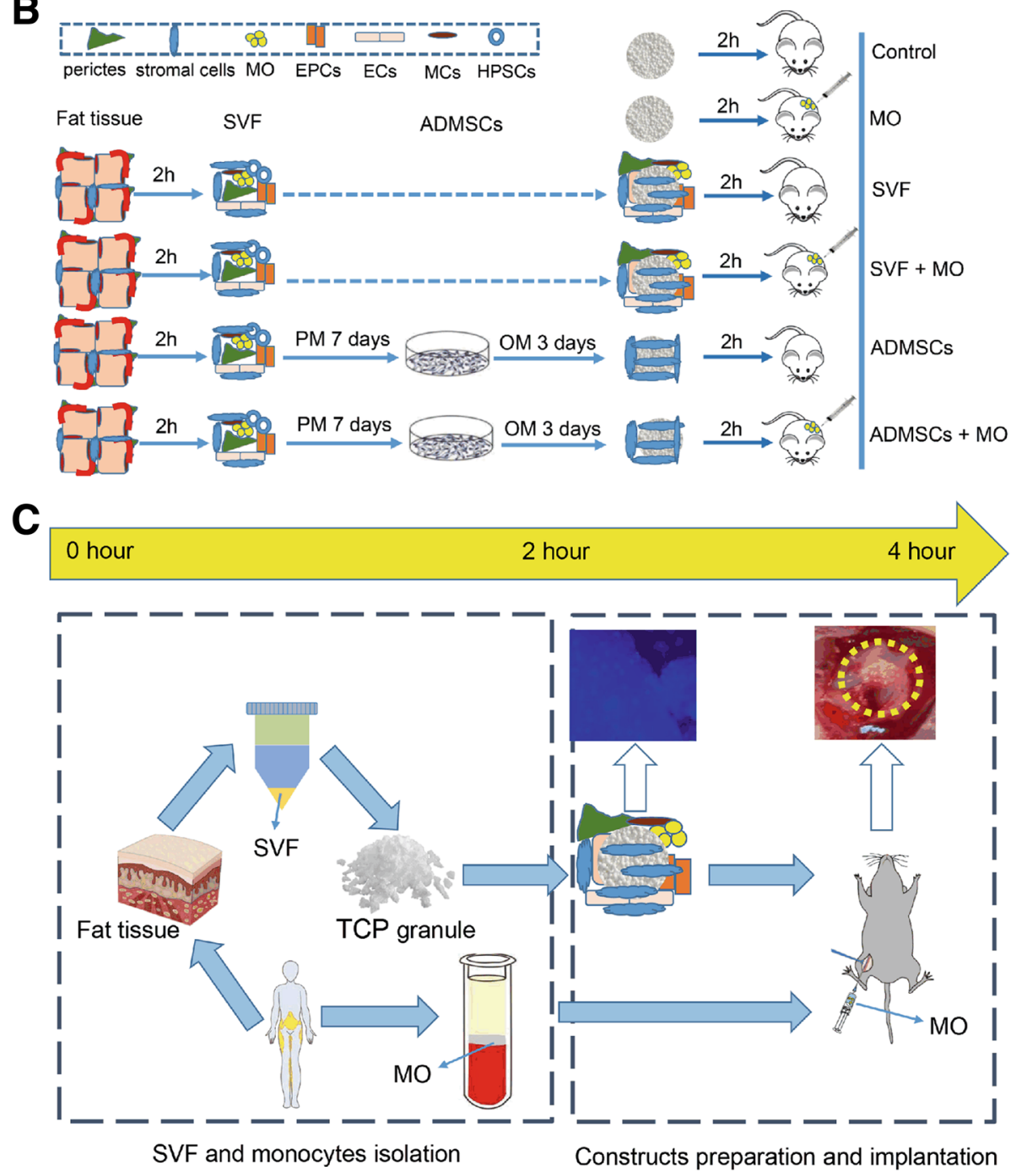

Fig. 1 The preparation of intraoperative and conventional approach-based bone constructs. a Cell type and number used for different construct preparation. b Schematic structure of the experimental design. PM, proliferation medium; OM, osteogenic medium; EPCs, endothelial progenitor cells; ECs, endothelial cells; MCs, mast cells; HPSCs, hematopoietic stem/progenitor cells. c Timeline toward to the intraoperative preparation of cell-based bone constructs. The intraoperative bone constructs can be made within $4 \mathrm{~h}$. Monocytes were added to the defect sites when bone constructs were put into femoral defects (yellow dashed circle) 
(Crl:NIH-Foxn1 ${ }^{\text {rnu }}$, Charles River) weighing between 250 and $300 \mathrm{~g}$ were used in the present study. All rats were pre-medicated with an intramuscular injection $(5 \mathrm{mg} /$ $\mathrm{kg}$ ) of carprofen. General anesthesia with nitrous oxide, oxygen, and isoflurane were applied during the surgery. Bone defects were surgically created $(3 \mathrm{~mm}$ diameter, $3 \mathrm{~mm}$ depth) bilaterally in the femoral condyles using a 3-mm burr (Hager \& Meisinger GmbH, Neuss, Germany) at low rational speed. MO $(n=14), \operatorname{SVF}(n=$ $16)$, SVF $(n=16)$, ADMSCs $(n=16)$, and ADMSCs+MO $(n=16)$ grafted implants were then placed into defects with acellular controls $(n=14)$. The soft tissue incision was then closed with superficial sutures. In all cases, the transplantation was performed within $4 \mathrm{~h}$. After surgery, $150 \mathrm{mg} / \mathrm{kg}$ buprenorphine (Temgesic, Schering-plough, Amstelveen, the Netherlands) was subcutaneously given for 2 days to reduce postoperative pain. Four and 10 weeks after implantation, all rats were sacrificed by inhalation of $\mathrm{CO}_{2}$. The constructs with surrounding tissue were harvested and fixed in a $4 \%$ formalin buffer for $48 \mathrm{~h}$. Samples were randomly selected and then dehydrated and embedded in paraffin for histochemistry and immunohistochemistry process. The rest of samples were randomly distributed, dehydrated, and embedded in polymethyl methacrylate (PMMA) for the histological process.

\section{Histological and histomorphometric analysis}

After fixation, certain specimens were dehydrated in a graded series of ethanol and embedded in PMMA without decalcification. Specimens were sectioned longitudinally $(335 \mu \mathrm{m}$ thick) using a diamond saw (Leica Microsystems SP 1600, Nussloch, Germany). Three sections representative of the center of the defect and subcutaneous sample from each specimen were then stained with methylene blue/basic fuchsin to evaluate new bone formation. Stained samples were further photographed with Zeiss Imager Z1 microscope equipped with the Axiocam camera using AxioVision 4.8 software. After that, the bone formation percentage (BF) was determined in stained sections using an automated image-analysis system (Leica Qwin Proimage analysis system, Wetzlar, Germany) which recognize bone tissue based on different RGB values from highly magnified images. Minor manual corrections were made to ensure the precise selection of newly formed bone in the defects. The percentage of new bone formation was measured by normalized to initial defect area measured from the cross section.

\section{Immunohistochemical and histochemical staining}

After fixation, samples from each group were decalcified in an EDTA solution, dehydrated in a series of alcohol and embedded in paraffin. The specimens were sectioned $(n=3)$ at a thickness of $5 \mu \mathrm{m}$ and stained with hematoxylin and eosin for conventional, qualitative bright field light microscopy analysis. Three additional sections per specimen were further stained with Masson's trichrome and observed by light microscopy. For immunostaining, paraffin sections were rehydrated in serials of ethanol and antigen was retrieved in sodium citrate buffer ( $\mathrm{PH} 6.0)$ at $70{ }^{\circ} \mathrm{C}$ for $10 \mathrm{~min}$. Subsequently, slides were blocked with $10 \%$ normal donkey serum (NDS) and then incubated with the primary antibody overnight at $4{ }^{\circ} \mathrm{C}$. Slides were then treated with a biotin-conjugated secondary antibody (Chemicon, Temecula, USA) for $1 \mathrm{~h}$ at room temperature, followed by counterstaining with hematoxylin. Negative controls using $2 \%$ NDS instead of the primary antibody were generated in parallel to ensure that the staining was specific. Finally, the sections were dehydrated and mounted. Stained sections were photographed with the same Zeiss Imager Z1 microscope. To detect human cells in the constructs implanted in rats, immunostaining was performed using human specific anti-mitochondria antibody (EMD Millipore, No. MAB1273, Amsterdam-Zuidoost; diluted 1:200) as protocol indicated. The number of human cells per construct was calculated by volumetrically relating slide volume to defect volume. Human and rat skin sections were used as positive and negative controls, respectively. The presence of human origin macrophages was assessed by using human specific mouse anti-human CD68 (Dako, Leuven, Belgium; diluted 1:200). To stain blood vessles, monoclonal antibody mouse anti- human/ rat $\alpha$-smooth muscle actin ( $\alpha$-SMA, Sigma-Aldrich, No. A2547, Billerica, USA; diluted 1:128,000) was used. For Tartrate-resistant acid phosphatase (TRAP) staining, sections were deparaffinized, rinsed in PBS and incubated with a solution containing $50 \mathrm{mM}$ sodium acetate (pH 5.2), $0.15 \%$ Naphtol-AS-TR-phosphate, $50 \mathrm{mM}$ sodium tartrate, and 0.1\% Fast Red T.R. (Sigma-Aldrich Chemie Gmbh, Taufkirchen, Germany) for 30-40 min at room temperature. Subsequently, the sections were rinsed in PBS and counterstained.

\section{Statistical analysis}

Cells from three donors were used for in vitro experiments and cells from donor 1 were used for in vivo donor-matched comparison. Data are presented as means \pm standard deviations (SD). Statistical analysis was performed using a one-way ANOVA with Graphpad Prism 5 software. When ANOVA indicated a significant difference between different groups, a Tukey Post-hoc Multiple Comparisons was performed. An unpaired Student's $t$ test was used to compare the calcium content between SVF and ADMSCs. $P$ values $<0.05$ were regarded as significant. 


\section{Results}

Comparative characterization of human ADMSCs and SVF Before construct preparation, we performed cytofluorimetric analysis to characterize SVF and ADMSCs respectively. The analysis of stromal cell markers (CD73, CD90, and CD105) showed consistent presence of stromal cells in SVF and stromal cells took up around one third of the SVF population (Additional file 1: Figure S1).

\section{Preparation of constructs and viability assessment}

To prepare SVF constructs, we seeded $3 \times 10^{6}$ SVF cells on $21 \mathrm{~mm}^{3}$ TCP granules and incubated these in proliferation medium for $2 \mathrm{~h}$ to allow for cell attachment. Similarly, we seeded $1 \times 10^{6}$ ADMSCs on TCP granules to obtain a comparable number of stromal cells on each construct. Subsequently, we added $1 \times 10^{6}$ monocytes to the SVF and ADMSC constructs in wells in vitro or to the constructs in the defects in vivo (Fig. 1a). Based on the design, from the isolation of SVF cells and peripheral blood monocytes till implantation of SVF constructs with monocytes, this procedure can be performed within $4 \mathrm{~h}$ (Fig. 1b, c). In contrast, the conventional ADMSC-based approach takes at least 10 days. To assess cell attachment to the prepared constructs, we performed nuclei and actin staining. Cells showed homogeneous distribution over the surface of granules (Additional file 2: Figure S2). Cell viability after $2 \mathrm{~h}$ in vitro incubation demonstrated that the majority of cells attached to the granules were viable, without apparent differences in dead cells between the experimental groups (Additional file 2: Figure S2).

\section{Monocytes promote osteogenic differentiation of SVF and ADMSCs}

To study cellular behavior upon effect of cell-cell interactions between monocytes and SVF or ADMSCs, we cultured cell-loaded constructs in osteogenic medium for up to 4 weeks and assessed cell proliferation (DNA content; Fig. 2a), osteogenic differentiation (ALP-activity; Fig. 2b), and mineralization (calcium assay; Fig. 2c). All cells on the granules showed proliferation over time. More specific, SVF and ADMSCs showed similar cell proliferation profiles (Fig. 2a). In contrast, cultures containing monocytes showed a significantly increased proliferation (Fig. 2a). Additionally, whilst monocyte monocultures did not show ALP activity, monocytes slightly increased ALP activity in co-cultures with either SVF or ADMSCs (Fig. 2b). Moreover, monocytes further significantly increased mineralization in co-cultures with either SVF $(\sim 2$-fold $)$ or ADMSCs $(\sim 1.5$-fold $)$ compared to SVF and ADMSCs monocultures in osteogenic medium, respectively (Fig. 2c). In contrast, all cultures

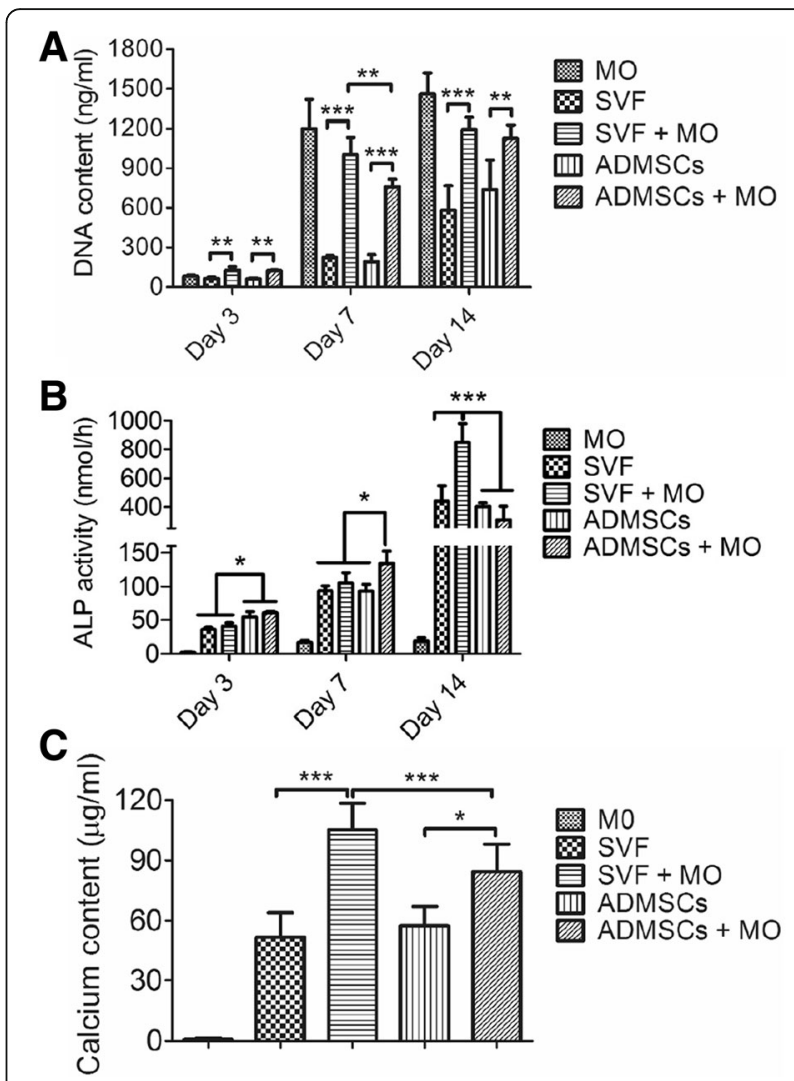

Fig. 2 In vitro cell behavior of SVF and ADMSCs upon co-culture with monocytes. a Cell proliferation of SVF, ADMSCs, and co-culture with monocytes quantified by DNA content $(n=4 ; * * *<0.001)$. b Osteogenic differentiation of SVF, ADMSCs, and co-culture with monocytes quantified by ALP activity $\left(n=4 ;{ }^{*}<0.05\right.$; $\left.{ }^{* * *}<0.001\right)$. c Mineralization of SVF, ADMSCs, and co-culture with monocytes after 4 weeks quantified by calcium content $\left(n=4\right.$; $^{*}<0.05$; $\left.{ }^{* *}<0.001\right)$

after seeding (day 0) and constructs cultured in proliferation medium (non-differentiation controls) had a negligible value for ALP activity and calcium content (data not shown).

\section{SVF cells and ADMSCs induce distinct polarization of monocytes/macrophages}

In parallel with our experiments focused on the effect of monocyte addition to either SVF or ADMSC cultures, we oppositely focused on effects of SVF or ADMSCs on monocytes activation and polarization. Therefore, we set up an in vitro culture model $[21,22]$ and analyzed macrophage polarization via cytokine expression profiles of the pro-inflammatory M1 macrophage cytokine TNF- $\alpha$ and the pro-wound healing M2 macrophage cytokine TGF- $\beta$ (Fig. 3a). Conventional induction into M1 macrophage polarization via LPS+IFN- $\gamma$ and into M2 macrophage polarization via IL-4+IL-13 stimulation was used as control. TCP granules used as cell carrier material in our experiments induced polarization into a 

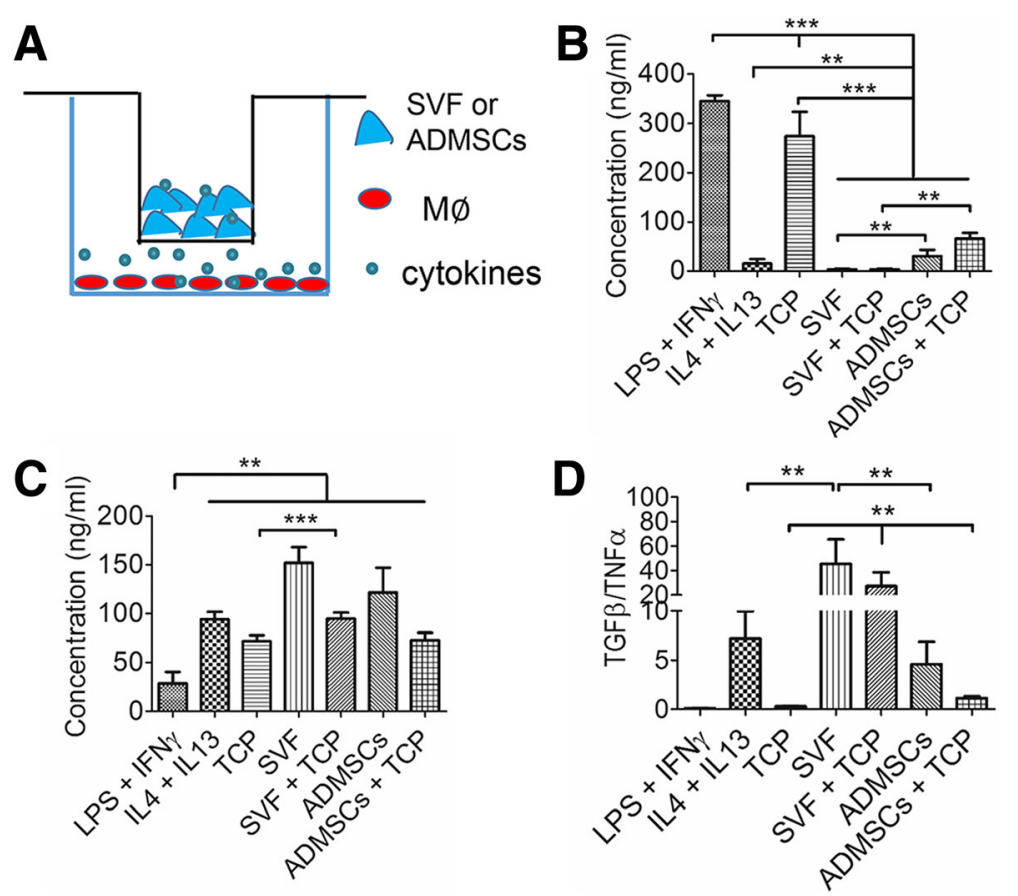

Fig. 3 In vitro cell behavior of monocytes upon co-culture with SVF or ADMSCs. a Schematic diagram of SVF and ADMSCs indirectly co-cultured with macrophages by a transwell. b Quantification of pro-inflammatory cytokine TNF-a in the co-culture medium $\left(n=6\right.$; ${ }^{* *} p<0.01$; $\left.{ }^{* * *}<0.001\right)$. $\mathbf{c}$ Quantification of anti-inflammatory cytokine TGF- $\beta$ in the co-culture medium $\left(n=6 ;{ }^{* *} p<0.01 ;{ }^{* * *}<0.001\right)$. d Ratio of TGF- $\beta /$ TNF- $a\left(n=6\right.$; ${ }^{* *} p<$ 0.01). LPS- and IFN- $\gamma$-induced M1 macrophages and IL-4- and IL-13-induced M2 macrophages were set as control respectively

pro-inflammatory M1 macrophage, while SVF and ADMSCs induced macrophage polarization toward the pro-wound healing M2 type (Fig. 3b, c). The ratio TGF- $\beta /$ TNF- $\alpha$ (Fig. 3d) clearly shows the overall M2 macrophage polarization stimulation, particularly by SVF.

\section{Cell-based constructs enhance early bone healing}

To assess the bone regeneration capacity, we implanted control, MO, SVF, SVF+MO, ADMSCs, and ADMSCs $+\mathrm{MO}$ constructs ( $n \geq 7$ for each group) into rat femoral defects (3 $\mathrm{mm}$ in diameter, $2.8 \mathrm{~mm}$ in depth) and evaluated bone regeneration histologically and histomorphometically after 4 and 10 weeks. Methylene blue/basic fuchsin staining (Fig. 4a) displayed substantial amounts of newly formed bone at the defect margins in between the voids of TCP granules in all SVF and ADMSC constructs after 4 weeks, irrespective of monocyte addition. In contrast, control and MO constructs showed limited amounts of newly formed bone. Histomorphometrically, SVF $(34.5 \pm 8.6 \%)$ showed similar amounts of new bone formation to ADMSCs $(27.1 \pm 6.0 \%)$, both of which regenerated approximately three times more bone compared to control and MO (Fig. 4b).

We further assessed the contribution of SVF and ADMSCs to bone formation using specific anti-human mitochondrial staining to detect human cells (i.e., SVF,
ADMSCs and monocytes) within the bone defect area (Fig. 4c and Additional file 3: Figure S3). SVF and SVF $+\mathrm{MO}$ constructs clearly showed the presence of human cells $(17,800 \pm 19,530$ and $5600 \pm 6460$ per construct respectively) in the vicinity of the TCP granulate and newly formed bone, but not within the newly formed bone. This positive staining for human cells originated from SVF, as we observed no positive staining for the human monocyte/macrophage marker CD68 (Additional file 4: Figure S4) nor for the osteoclastic marker TRAP (Additional file 5: Figure S5). In contrast, ADMSCs and ADMSCs+MO constructs did not show any human cells within histological sections. In addition, SVF and ADMSCs constructs showed apparently larger vessel-like structures compared to control and MO constructs, which suggests beneficial effects of SVF and ADMSCs on the vascularization of implanted constructs (Fig. 4d).

\section{SVF constructs show complete bone defect healing}

At 10 weeks post implantation, SVF constructs (w/$\mathrm{MO}$ ) exhibited complete healing of the defect site with newly formed bone present throughout the entire defect area (Fig. 5a). In contrast, ADMSCs (w/- MO) showed substantial bone ingrowth centripetally from the original defect edge toward the defect center without presence of newly formed bone in the center. Control and $\mathrm{MO}$ 
A

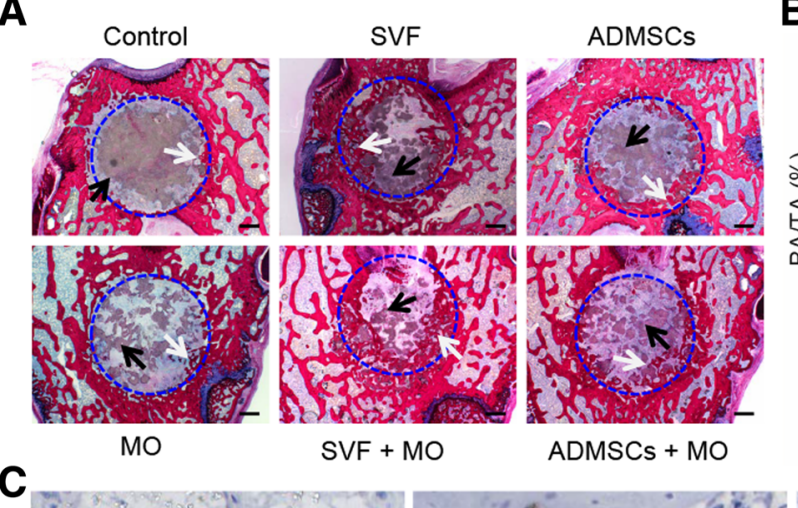

B
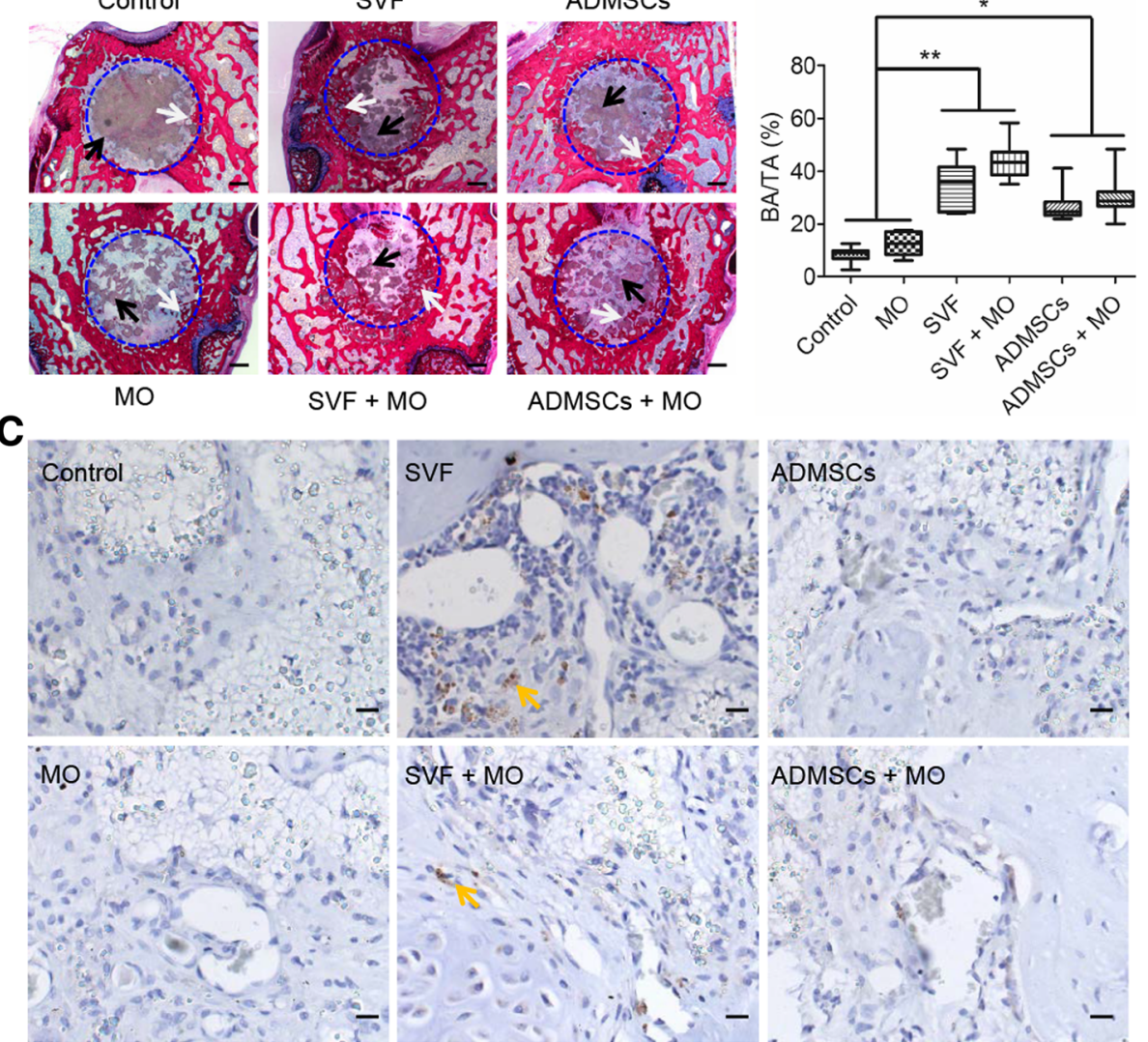
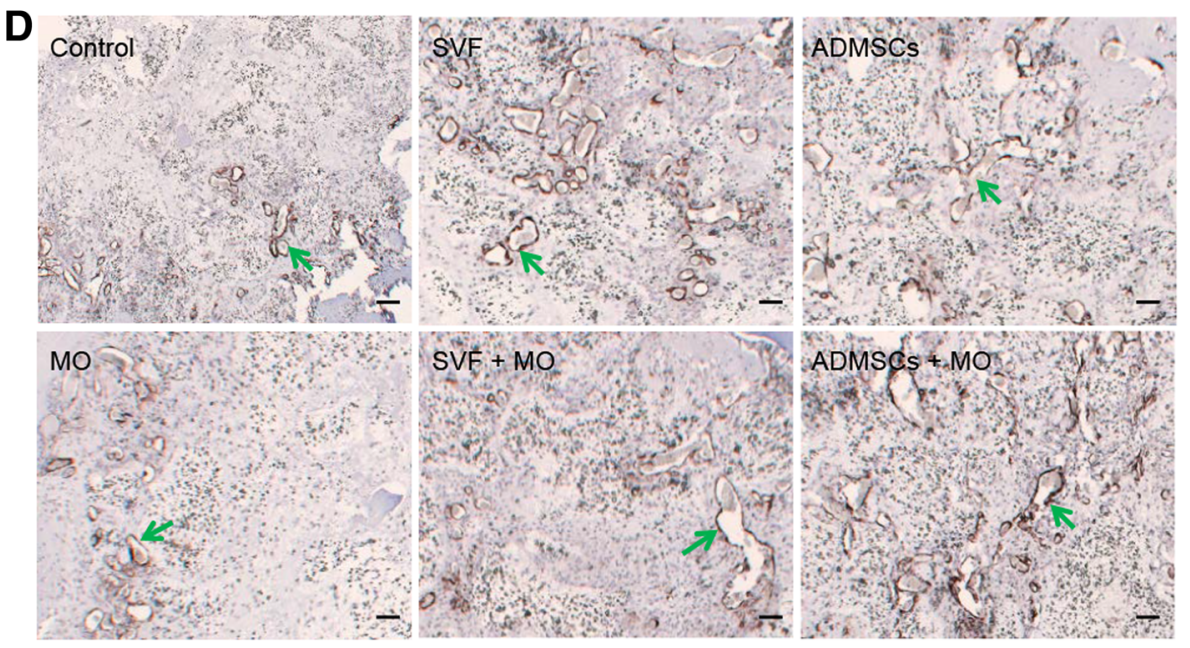

Fig. 4 New bone quantification and representative images of histology and immunohistochemistry staining after 4 weeks implantation in the femoral condyle bone defect. a Representative histological sections (methylene blue/basic fuchsin). Blue dashed circles indicate original bone defect, black arrows indicate TCP granulate, and white arrows indicate newly formed bone; scale bar $=500 \mu \mathrm{m}$. $\mathbf{b}$ Histomorphometric analysis of new bone area (BA) within the total defect area (TA; $n=6$ to $\left.7 ;{ }^{*} p<0.05 ;{ }^{* *}<0.01\right)$. c Representative images of anti-human mitochondria staining. Yellow arrows indicate positive staining. $\mathbf{d}$ Representative images of anti-a-SMA staining. Green arrows indicate blood vessels; scale bar $=10 \mu \mathrm{m}$

constructs still showed large defect areas containing fibrous connective tissue with only sparse bone formation at the edge of the original defect. Residual TCP granulate was still present in non-healing defects and integrated with new bone in SVF and ADMSCs constructs.
Quantitative analysis of new bone formation by histomorphometry (Fig. 5b) revealed superior amounts of newly form bone for SVF and SVF+MO constructs $(64.9 \pm 9.1 \%$ and $66.3 \pm 6.0 \%$, respectively) compared to ADMSCs (45.4 $\pm 3.7 \%), A D M S C s+M O(55.2 \pm 10.6 \%)$, 


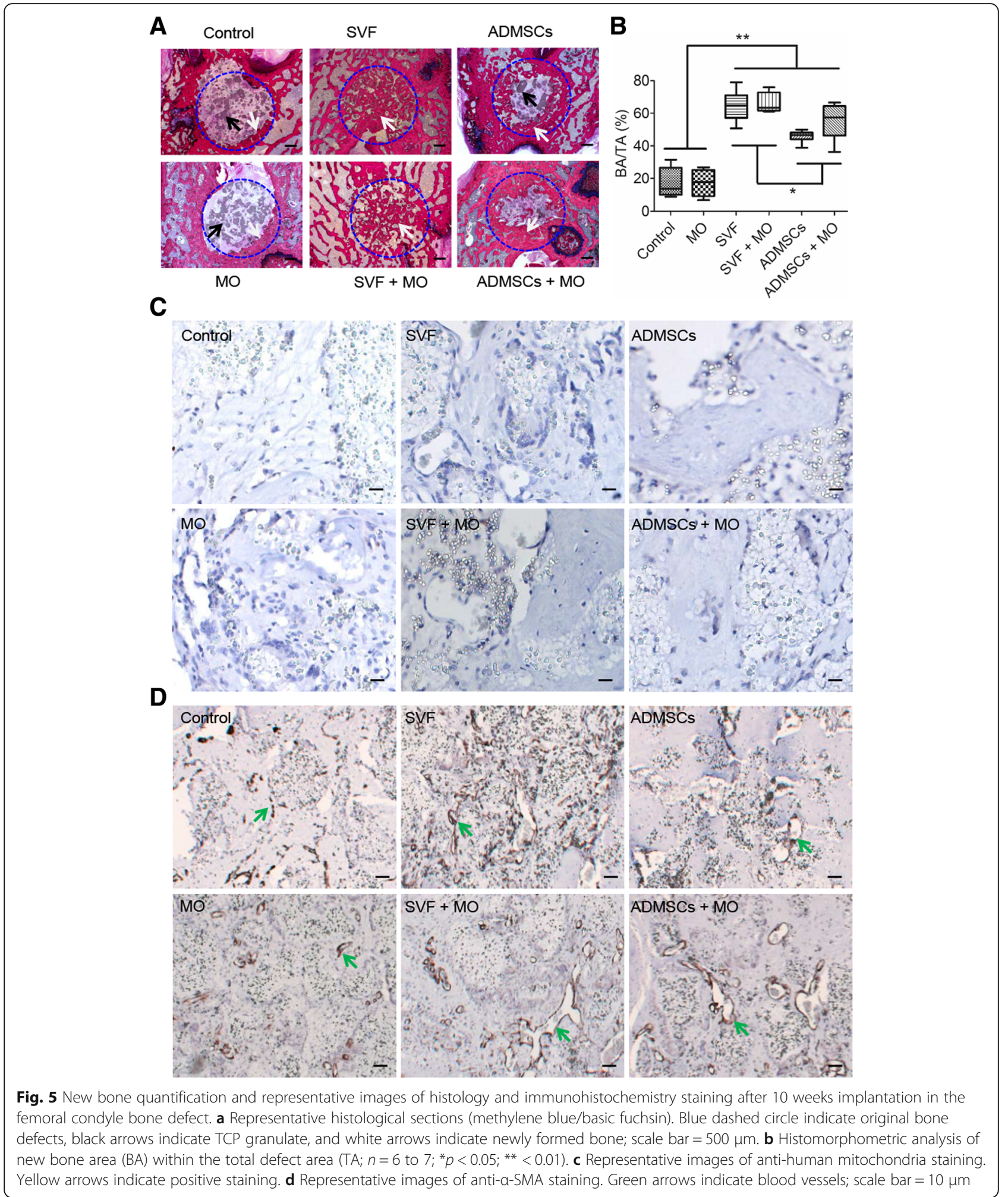

MO (17.3 $\pm 8.0 \%)$, and Control $(17.2 \pm 9.0 \%)$ constructs. Further, ADMSC and ADMSC+MO constructs showed significantly higher bone formation compared to $\mathrm{MO}$ and control constructs.
Anti-human mitochondrial staining to detect cells of human origin demonstrated absence of human cells for any type of implanted construct after 10 weeks (Fig. 5c). Based on $\alpha$-SMA staining to assess vascularization, 
constructs with SVF or ADMSCs apparently gained more organized and elongated vessel-like structures (Fig. 5d). Different to our observations after 4 weeks of implantation, osteoclastic activity was abundantly present in all defects containing cell-based constructs and only to a limited extent in control constructs (Additional file 5: Figure S5).

\section{Discussion}

Cell-based bone tissue engineering, especially with the application of bone marrow-derived MSCs, represents an appealing alternative for the current best practice treatments using auto-/allografts for bone regeneration. However, this approach is challenged by complex, impractical and expensive in vitro cell expansion due to the low numbers of MSCs in the bone marrow. Aiming to circumvent these, we followed an intraoperative approach based on stromal vascular fraction (SVF) from fat tissue, as described recently [13, 14]. However, the efficacy of this intraoperative approach has not been directly compared to conventional ADMSC-based method with cells of the same donor. As another type of easy access cells with potential stimulatory effects on wound healing and stromal cell differentiation [17, 18], we explored the addition of monocytes to both ADMSC and SVF constructs both in vitro and in vivo using a rat femoral condyle bone defect model.

SVF is reported to be a heterogeneous mixture of MSCs, endothelial progenitor cells, pericytes, mast cells, pre-adipocytes, and other cell types [7, 11]. From flow cytometry and in vitro cell culture results, we proved that MSCs take up around one third of the whole SVF population, which corroborates previous reports [2325]. As MSCs were designated as the main effective cells in bone formation, we used 3 million SVF cells versus 1 million purified ADMSCs, which were isolated from the same donor during the same procedure. We then compared their osteogenic potential in vitro and bone regeneration capacity in vivo. In vitro mineralization demonstrated a similar osteogenic potential between SVF and ADMSCs. In contrast, although both SVF and ADMSCs improved bone defect healing compared to cell-free controls, data from our in vivo work demonstrated superiority of SVF constructs over ADMSC constructs regarding bone-regenerative capacity. This difference between in vitro and in vivo results suggests that in vitro osteogenic differentiation capacity is not predictive for in vivo bone healing capacity. The synergetic effect of endothelial progenitor cells and pericytes contained in SVF probably plays an important role in the superiority of SVF bone healing [24, 26]. Important observations, likely related to the superior bone regeneration for SVF and SVF + MO constructs, were the prolonged presence of SVF cells in the bone defect region compared to ADMSCs in spite of in low numbers (less than $2 \%$ of seeded cells in the constructs after 4 weeks implantation). The lower number of human cells in SVF $+\mathrm{MO}$ compared to SVF likely resulted from the low survival and proliferation rates of seeded SVF cells subjected to more stringent inflammation due to the addition of monocytes [27-29].

Speculating about the mechanism of SVF- and ADMSCs-based bone regeneration, our results and those of others [30] indicate that direct differentiation of grafted cells into bone forming osteoblasts or eventually embedded osteocytes seems very unlikely. Further, it has been postulated that the grafted cells provide osteoinductive signals or act as a source of trophic factors to modulate microenvironments [31-33]. Here, we did not observe grafted human cells within the newly formed bone (only in the vicinity at 4 weeks), ruling out the mechanism of direct differentiation of SVF or ADMSCs into bone forming osteoblasts or osteocytes. Consequently, we speculate that SVF and ADMSCs exerted a certain paracrine effect on surrounding host cells to stimulate bone regeneration. The exact paracrine factors secreted by these implanted cells responsible for bone healing are largely unknown yet due to the complexity of in vivo conditions. Still, few pioneering reports indicated several possible roles of grafted cells, such as promoting progenitor cells migration [34, 35], benefiting vascularization $[31,36]$, and modulating immune response [37, 38], to stimulate bone regeneration. Amongst these hypotheses, the immunomodulatory role by MSCs in tissue regeneration recently gained much attention. MSCs have been shown to modulate the function of both innate and adaptive immune cells through direct interactions as well as releasing numerous bioactive soluble factors $[39,40]$. In particular, MSCs can directly polarize naïve macrophages toward the M2 phenotype to exert a therapeutic effect on skin, brain, and muscle [41-43]. In bone regeneration process, M2 phenotype macrophages can promote tissue remodeling by releasing cytokines such as basic fibroblast growth factor (bFGF) to benefit the host vascularization [44] and increase osteogenesis by releasing certain growth factors such as OSM and BMP-2 [45]. In the present study, SVF and ADMSCs activated and polarized monocytes/macrophages into M2 phenotype as proved in in vitro co-culture experiment. These M2 macrophages likely further led to more profound vascularization as our results and previous studies reported [24] and subsequent more bone formation compared to constructs without SVF and ADMSCs in vivo. The coincidence of superior immunoregulatory effect and more remarkable bone regeneration capacity by grafted SVF further support the hypothesis about immunoregulatory roles in bone healing. Nonetheless, this warrants further 
investigations into the paracrine factors underlying, such as recently reported extracellular vesicles route $[46,47]$.

Due to their reported beneficial effects on osteogenic differentiation $[17,18]$ and the easy access via a venous puncture, the concept of harnessing the power of monocytes/macrophages for bone tissue engineering was newly proposed $[48,49]$. However, it has never been applied for cell-based bone regeneration. Our study also confirmed their beneficial effects on in vitro osteogenic differentiation, evidenced by significantly higher ALP activity and mineralization in co-cultures with SVF or ADMSCs. Interestingly, monocytes exerted a more obvious effect on SVF compared to ADMSCs, which suggests differences in cell status (undifferentiated versus differentiated), cell components (heterogeneous and homogenous), and cell-cell interactions of SVF compared to ADMSCs. Nonetheless, in our orthotopic animal model, we did not observe this favorable effect on bone regeneration following monocyte addition. As we did not observe specific immunostaining for human monocytes/macrophages in any of our histological sections (Additional file 3: Figure S3), this might be explained by the short duration of exogenously added monocytes present in the defects, the relatively limited half-live of this cell life, and less adhesive properties compared to stromal cells $[50,51]$. However, given the newly reported pivotal roles of immune cells, including monocytes, macrophages, NK cells, and lymphocytes in tissue regeneration $[49,52,53]$, introduction of immune cells in cell-based bone tissue engineering still seems an attractive treatment strategy for bone regeneration.

Our study also has several limitations. First, we used human monocytes from a cell line rather than human primary monocytes considering the experimental feasibility and reproducibility. Although THP-1 has been proven as a suitable cell culture model for primary monocytes [54], further studies are needed to analyze the behavior of SVF and ADMSCs co-cultured with primary monocytes, especially when using monocytes and SVF derived from the same patient. Secondly, SVF and ADMSC constructs enhanced bone formation after 4 weeks, which suggests that the seeded cells function in the early post-implantation stage. Therefore, observation of host response (i.e., cell infiltration, vascularization, and macrophage phenotype) at earlier time points would likely provide more clarity on the potential immunoregulatory role of grafted cells in bone healing. In addition, due to the component similarity and close distribution between TCP and newly formed bone, it is not practical to use regular X-ray or micro-CT to provide additional information on bone density and quality.

Above all, instead of merely focusing on the outcome of cell-based bone tissue engineering as in most studies, we emphasized particularly on the potential of cell-based constructs for clinical applications. Since both SVF and monocytes can be freshly isolated via standard procedures during the surgical procedure, this strategy meets the requirements of "good manufacturing practice" and "during the same visit" defined by the concept of intraoperative preparation. In addition, this method likely reduces the high-cost associated to ex vivo cell expansion and its safety was confirmed in various clinical applications [13, 55]. Therefore, this 'easy access cells' based intraoperative approach and its superior bone-regenerative capacity will greatly aid the concept, design, development, and practicality of cell-based bone tissue engineering.

\section{Conclusion}

To conclude, this study described the feasibility of a clinically compliant method to intraoperatively generate bone constructs by using easy access cells, namely stromal vascular fraction (SVF) from adipose tissue and monocytes from the peripheral blood. Our data demonstrated the equal osteogenic potential of SVF as expanded ADMSCs in vitro, which for both was significantly enhanced upon co-culture with monocytes. When applied to bone defects, SVF demonstrated a significantly higher bone formation compared to constructs grafted with ADMSCs and cell-free controls. This was further indicated attributing to the distinct differences of immunoregulatory effects between SVF and ADMSCs on macrophages. Given the superior bone regeneration capacity of SVF and the easy access of SVF and monocytes during the surgical procedure, the strategy proposed in this study has great potential in the clinic to develop new cell-based bone products to treat damaged bone.

\section{Additional files}

\footnotetext{
Additional file 1: Figure S1. Cell characterization of SVF and ADMSCS by flow cytometry. SVF and ADMSCs were stained with APC-conjugated CD73, PE-Cy5-conjugated CD90, PE-conjugated CD105 and FITCconjugated CD45 and then sorted by flow cytometry. (TIF 7284 kb)

Additional file 2: Figure S2. Cell attachment and viability on TCP granules after seeding for $2 \mathrm{~h}$. For the attachment, cells were stained with DAPI for the nuclei (blue) and Alexa Fluor 568 conjuncted phalloidin for the actin (red); scale bar $=500 \mu \mathrm{m}$. For the viability, cells were stained with calcein-AM (green) and ethidium homodimer-1 (red). White arrows indicate live cells and red arrows indicate dead cells; scale bar $=500 \mu \mathrm{m}$. (TIF 10203 kb)

Additional file 3: Figure S3. Representative images of anti-human mitochondria staining after 4 weeks orthotopic implantation. (A) SVF constructs at low magnification; Scale bar $=500 \mu \mathrm{m}$. (B) SVF constructs at high magnification. NB indicates new bone and TCP indicates TCP granules. Brown arrows indicate human origin cells. Scale bar $=100 \mu \mathrm{m}$. (C) SVF+MO constructs at low magnification; Scale bar $=500 \mu \mathrm{m}$. (D) SVF $+\mathrm{MO}$ constructs at high magnification. NB indicates new bone and TCP indicates TCP granules. Brown arrows indicate human origin cells; scale bar $=100 \mu \mathrm{m}$. (TIF $5613 \mathrm{~kb}$ )
} 
Additional file 4: Figure S4. Representative images of anti-human CD68 immunohistochemistry staining after 4 weeks orthotopic implantation. Black arrow indicates TCP granules. Yellow arrow indicates presence of human macrophages in the samples. PC indicates the positive control samples stained with anti-human CD68; Scale bar = $100 \mu \mathrm{m}$. (TIF $3236 \mathrm{~kb}$ )

Additional file 5: Figure S5. Representative images of TRAP immunohistochemistry staining after (A) 4 and (B) 10 weeks orthotopic implantation. Blue arrows indicate TRAP-positive signals in the defect region; bar $=500 \mu \mathrm{m}$. (TIF $9162 \mathrm{~kb}$ )

\section{Abbreviations}

ADMSCs: Adipose tissue mesenchymal stem cells; SVF: Stromal vascular fraction; MSCs: Mesenchymal stromal cells; MO: Monocytes; TRAP: Tartrateresistant acid phosphatase; TCP: Tricalcium phosphate; BSA: Bovine serum albumin; PMA: Phorbol myristate acetate; LPS: Lipopolysachariden; IL4: Interlukine-4; IL-13: Interlukine-13; IFN-ү: Interferon- $\gamma$; TNF-a: Tumor necrosis factor-a; TGF- $\beta$ : Transforming growth factor- $\beta$; ALP: Alkaline phosphatase; bFGF: Basic fibroblast growth factor; DAPI: 4',6-Diamidino-2phenylindole

\section{Acknowledgements}

We acknowledge Natasja van Dijk for technical assistance with cross sections making and immunostaining performance.

\section{Funding}

This study gets financial support from Dutch ZonMw GameChanger Project 'ioCOMPONENTS' (No. 40-41400-98-16031) and China Scholarship Council (No. 2013622061).

\section{Availability of data and materials}

All data generated and/or analyzed during this study are included in this published article and its additional files.

\section{Authors' contributions}

YZ designed and conducted the study, acquired and analyzed all data, and wrote the manuscript; ECG and WAC participated in the animal surgery, sample retrieval, and histomorphometrical analysis. HT and AMPM participated in the cell culture and cell seeding process. JJJPvdB gave the concept and designed the whole study including animal experiment, conducted the animal surgery, and participated in the interpretation of the data and writing and final approval of the manuscript. All authors read and approved the final manuscript.

\section{Ethics approval}

Animal study was conducted according to Dutch national guidelines for use and care of laboratory animals after ethical approval by the Centrale Commissie Dierproeven (CCD; project 2015-137). This study was performed according to an informed protocol for handling human tissue approved by the Dutch Central Committee on Human Research (Commissie Mensgebonden Onderzoek; dossier number \#3252).

Informed consent was obtained from all participants that donated fat tissue from Radboudumc.

\section{Consent for publication}

Not applicable.

\section{Competing interests}

The authors declare that they have no competing interests.

\section{Publisher's Note}

Springer Nature remains neutral with regard to jurisdictional claims in published maps and institutional affiliations.

\section{Author details}

'Department of Biomaterials, PO Box 9101, 6500HB Radboudumc, Nijmegen, the Netherlands. ${ }^{2}$ Department of Plastic Surgery, Tongji Hospital, Huazhong University of Science and Technology, Wuhan 430074, China. ${ }^{3}$ Department of Biosciences, Federal University of São Paulo, Santos, São Paulo, Brazil.
Received: 22 May 2018 Revised: 16 August 2018

Accepted: 30 September 2018 Published online: 25 October 2018

\section{References}

1. Betz RR. Limitations of autograft and allograft: new synthetic solutions. Orthopedics. 2002;25(5):S561-70.

2. Quarto R, Mastrogiacomo M, Cancedda R, Kutepov SM, Mukhachev V, Lavroukov A, Kon E, Marcacci M. Repair of large bone defects with the use of autologous bone marrow stromal cells. N Engl J Med. 2001;344(5):385-6.

3. Caplan Al. Mesenchymal stem cells. J Orthop Res. 1991;9(5):641-50.

4. Ma J, Both SK, Yang F, Cui FZ, Pan J, Meijer GJ, Jansen JA, van den Beucken JJ. Concise review: cell-based strategies in bone tissue engineering and regenerative medicine. Stem Cells Transl Med. 2014:3(1):98-107.

5. Sakaguchi Y, Sekiya I, Yagishita K, Muneta T. Comparison of human stem cells derived from various mesenchymal tissues: superiority of synovium as a cell source. Arthritis Rheum. 2005;52(8):2521-9.

6. Zuk PA, Zhu M, Ashjian P, De Ugarte DA, Huang Jl, Mizuno H, Alfonso ZC, Fraser JK, Benhaim P, Hedrick MH. Human adipose tissue is a source of multipotent stem cells. Mol Biol Cell. 2002;13(12):4279-95.

7. Zuk PA, Zhu M, Mizuno H, Huang J, Futrell JW, Katz AJ, Benhaim P, Lorenz $H P$, Hedrick MH. Multilineage cells from human adipose tissue: implications for cell-based therapies. Tissue Eng. 2001:7(2):211-28.

8. Yoon E, Dhar S, Chun DE, Gharibjanian NA, Evans GR. In vivo osteogenic potential of human adipose-derived stem cells/poly lactide-co-glycolic acid constructs for bone regeneration in a rat critical-sized calvarial defect model. Tissue Eng. 2007:13(3):619-27.

9. Niemeyer P, Fechner K, Milz S, Richter W, Suedkamp NP, Mehlhorn AT, Pearce S, Kasten P. Comparison of mesenchymal stem cells from bone marrow and adipose tissue for bone regeneration in a critical size defect of the sheep tibia and the influence of platelet-rich plasma. Biomaterials. 2010; 31(13):3572-9.

10. Oedayrajsingh-Varma M, Van Ham S, Knippenberg M, Helder M, KleinNulend J, Schouten T, Ritt M, Van Milligen F. Adipose tissue-derived mesenchymal stem cell yield and growth characteristics are affected by the tissue-harvesting procedure. Cytotherapy. 2006;8(2):166-77.

11. Varma MJO, Breuls RG, Schouten TE, Jurgens WJ, Bontkes HJ, Schuurhuis GJ, Ham SMV, Milligen FJV. Phenotypical and functional characterization of freshly isolated adipose tissue-derived stem cells. Stem Cells Dev. 2007;16(1):91-104.

12. Prins H-J, Schulten EA, Ten Bruggenkate CM, Klein-Nulend J, Helder MN. Bone regeneration using the freshly isolated autologous stromal vascular fraction of adipose tissue in combination with calcium phosphate ceramics. Stem Cells Transl Med. 2016;5(10):1362-74.

13. Saxer F, Scherberich A, Todorov A, Studer P, Miot S, Schreiner S, Güven S, Tchang LA, Haug M, Heberer M. Implantation of stromal vascular fraction progenitors at bone fracture sites: from a rat model to a first-in-man study. Stem Cells. 2016:34(12):2956-66.

14. Todorov A, Kreutz M, Haumer A, Scotti C, Barbero A, Bourgine PE, Scherberich A, Jaquiery C, Martin I. Fat-derived stromal vascular fraction cells enhance the bone-forming capacity of devitalized engineered hypertrophic cartilage matrix. Stem Cells Transl Med. 2016;5(12):1684-94.

15. Inglis S, Christensen D, Wilson DI, Kanczler JM, Oreffo RO. Human endothelial and foetal femur-derived stem cell co-cultures modulate osteogenesis and angiogenesis. Stem Cell Res Ther. 2016;7(1):13.

16. Ma J, Beucken JJ, Both SK, Prins HJ, Helder MN, Yang F, Jansen JA Osteogenic capacity of human BM-MSCs, AT-MSCs and their co-cultures using HUVECs in FBS and PL supplemented media. J Tissue Eng Regen Med. 2015;9(7):779-88.

17. Nicolaidou V, Wong MM, Redpath AN, Ersek A, Baban DF, Williams LM, Cope AP, Horwood NJ. Monocytes induce STAT3 activation in human mesenchymal stem cells to promote osteoblast formation. PLoS One. 2012;7(7):e39871.

18. Pirraco RP, Reis R, Marques A. Effect of monocytes/macrophages on the early osteogenic differentiation of hBMSCs. J Tissue Eng Regen Med. 2013; 7(5):392-400.

19. Ma J, Both SK, Ji W, Yang F, Prins HJ, Helder MN, Pan J, Cui FZ, Jansen JA, van den Beucken JJ. Adipose tissue-derived mesenchymal stem cells as monocultures or cocultures with human umbilical vein endothelial cells: performance in vitro and in rat cranial defects. J Biomed Mater Res A. 2014; 102(4):1026-36.

20. Thirumala S, Gimble JM, Devireddy RV. Cryopreservation of stromal vascular fraction of adipose tissue in a serum-free freezing medium. J Tissue Eng Regen Med. 2010;4(3):224-32. 
21. Cho D, Kim MR, Jeong $H$, Jeong HC, Jeong MH, Yoon SH, Kim YS, Ahn Y. Mesenchymal stem cells reciprocally regulate the M1/M2 balance in mouse bone marrow-derived macrophages. Exp Mol Med. 2014;46(1):e70.

22. Kubosch EJ, Heidt E, Bernstein A, Böttiger K, Schmal H. The trans-well coculture of human synovial mesenchymal stem cells with chondrocytes leads to self-organization, chondrogenic differentiation, and secretion of TGF $\beta$. Stem Cell Res Ther. 2016;7(1):64

23. Zimmerlin L, Donnenberg VS, Pfeifer ME, Meyer EM, Péault B, Rubin JP, Donnenberg AD. Stromal vascular progenitors in adult human adipose tissue. Cytometry Part A. 2010;77(1):22-30.

24. Scherberich A, Galli R, Jaquiery C, Farhadi J, Martin I. Three-dimensional perfusion culture of human adipose tissue-derived endothelial and osteoblastic progenitors generates osteogenic constructs with intrinsic vascularization capacity. Stem Cells. 2007;25(7):1823-9.

25. Güven S, Mehrkens A, Saxer F, Schaefer DJ, Martinetti R, Martin I, Scherberich A. Engineering of large osteogenic grafts with rapid engraftment capacity using mesenchymal and endothelial progenitors from human adipose tissue. Biomaterials. 2011;32(25):5801-9.

26. Tawonsawatruk T, West C, Murray I, Soo C, Péault B, Simpson A. Adipose derived pericytes rescue fractures from a failure of healing-non-union. Sci Rep. 2016;6:22779.

27. Baldari S, Di Rocco G, Piccoli M, Pozzobon M, Muraca M, Toietta G. Challenges and strategies for improving the regenerative effects of mesenchymal stromal cell-based therapies. Int J Mol Sci. 2017;18(10):2087.

28. Copland IB, Galipeau J. Death and inflammation following somatic cell transplantation. Semin Immunopathol. 2011;33(6):535-50.

29. Martino MM, Maruyama K, Kuhn GA, Satoh T, Takeuchi O, Müller R, Akira S. Inhibition of IL-1R1/MyD88 signalling promotes mesenchymal stem celldriven tissue regeneration. Nat Commun. 2016;7:11051.

30. Gamblin AL, Brennan MA, Renaud A, Yagita H, Lézot F, Heymann D, Triche $V$, Layrolle P. Bone tissue formation with human mesenchymal stem cells and biphasic calcium phosphate ceramics: the local implication of osteoclasts and macrophages. Biomaterials. 2014;35(36):9660-7.

31. Todeschi MR, El Backly R, Capelli C, Daga A, Patrone E, Introna M, Cancedda R, Mastrogiacomo M. Transplanted umbilical cord mesenchymal stem cells modify the in vivo microenvironment enhancing angiogenesis and leading to bone regeneration. Stem Cells Dev. 2015;24(13):1570-81.

32. Czekanska EM, Ralphs JR, Alini M, Stoddart M. Enhancing inflammatory and chemotactic signals to regulate bone regeneration. Eur Cell Mater. 2014;28: 320-34.

33. Linero I, Chaparro O. Paracrine effect of mesenchymal stem cells derived from human adipose tissue in bone regeneration. PLoS One. 2014;9(9):e107001.

34. Lyons FG, Al-Munajjed AA, Kieran SM, Toner ME, Murphy CM, Duffy GP, O'Brien FJ. The healing of bony defects by cell-free collagen-based scaffolds compared to stem cell-seeded tissue engineered constructs. Biomaterials. 2010;31(35):9232-43

35. Seebach E, Freischmidt H, Holschbach J, Fellenberg J, Richter W. Mesenchymal stroma cells trigger early attraction of M1 macrophages and endothelial cells into fibrin hydrogels, stimulating long bone healing without long-term engraftment. Acta Biomater. 2014;10(11):4730-41.

36. Spiller KL, Freytes DO, Vunjak-Novakovic G. Macrophages modulate engineered human tissues for enhanced vascularization and healing. Ann Biomed Eng. 2015;43(3):616-27.

37. Kovach TK, Dighe AS, Lobo PI, Cui Q. Interactions between MSCs and immune cells: implications for bone healing. J Immunol Res. 2015;2015: 752510

38. Tasso R, Ulivi V, Reverberi D, Lo Sicco C, Descalzi F, Cancedda R. In vivo implanted bone marrow-derived mesenchymal stem cells trigger a cascade of cellular events leading to the formation of an ectopic bone regenerative niche. Stem Cells Dev. 2013;22(24):3178-91.

39. Le Blanc K, Mougiakakos D. Multipotent mesenchymal stromal cells and the innate immune system. Nat Rev Immunol. 2012;12(5):383.

40. Insausti CL, Blanquer M, García-Hernández AM, Castellanos G, Moraleda JM. Amniotic membrane-derived stem cells: immunomodulatory properties and potential clinical application. Stem Cells Cloning. 2014;7:53.

41. Aurora $A B$, Olson EN. Immune modulation of stem cells and regeneration. Cell Stem Cell. 2014;15(1):14-25.

42. Qiu X, Liu S, Zhang H, Zhu B, Su Y, Zheng C, Tian R, Wang M, Kuang H, Zhao X. Mesenchymal stem cells and extracellular matrix scaffold promote muscle regeneration by synergistically regulating macrophage polarization toward the M2 phenotype. Stem Cell Res Ther. 2018;9(1):88.
43. Yang Z, He C, He J, Chu J, Liu H, Deng X. Curcumin-mediated bone marrow mesenchymal stem cell sheets create a favorable immune microenvironment for adult full-thickness cutaneous wound healing. Stem Cell Res Ther. 2018;9(1):21.

44. Jetten N, Verbruggen S, Gijbels MJ, Post MJ, De Winther MP, Donners MM. Anti-inflammatory $M 2$, but not pro-inflammatory $M 1$ macrophages promote angiogenesis in vivo. Angiogenesis. 2014;17(1):109-18.

45. Zhang Y, Böse T, Unger RE, Jansen JA, Kirkpatrick CJ, van den Beucken JJ. Macrophage type modulates osteogenic differentiation of adipose tissue MSCs. Cell Tissue Res. 2017;369(2):273-86.

46. Chen B, Li Q, Zhao B, Wang Y. Stem cell-derived extracellular vesicles as a novel potential therapeutic tool for tissue repair. Stem Cells Transl Med. 2017;6(9):1753-8.

47. Keshtkar S, Azarpira N, Ghahremani MH. Mesenchymal stem cell-derived extracellular vesicles: novel frontiers in regenerative medicine. Stem Cell Res Ther. 2018:9(1):63.

48. Chen Z, Klein T, Murray RZ, Crawford R, Chang J, Wu C, Xiao Y. Osteoimmunomodulation for the development of advanced bone biomaterials. Mater Today. 2016;19(6):304-21.

49. Julier Z, Park AJ, Briquez PS, Martino MM. Promoting tissue regeneration by modulating the immune system. Acta Biomater. 2017;53:13-28.

50. Swirski FK, Pittet MJ, Kircher MF, Aikawa E, Jaffer FA, Libby P, Weissleder R. Monocyte accumulation in mouse atherogenesis is progressive and proportional to extent of disease. Proc Natl Acad Sci. 2006;103(27):10340-5.

51. Yona S, Jung S. Monocytes: subsets, origins, fates and functions. Curr Opin Hematol. 2010;17(1):53-9.

52. Liu Y, Wang L, Kikuiri T, Akiyama K, Chen C, Xu X, Yang R, Chen W, Wang S, Shi $S$. Mesenchymal stem cell-based tissue regeneration is governed by recipient T lymphocytes via IFN- $\gamma$ and TNF-a. Nat Med. 2011;17(12):1594.

53. Tosello-Trampont A, Surette FA, Ewald SE, Hahn YS. Immunoregulatory role of NK cells in tissue inflammation and regeneration. Front Immunol. 2017:8:301.

54. Chanput W, Mes JJ, Wichers HJ. THP-1 cell line: an in vitro cell model for immune modulation approach. Int Immunopharmacol. 2014;23(1):37-45.

55. Pak J, Chang J-J, Lee JH, Lee SH. Safety reporting on implantation of autologous adipose tissue-derived stem cells with platelet-rich plasma into human articular joints. BMC Musculoskelet Disord. 2013;14(1):337.

\section{Ready to submit your research? Choose BMC and benefit from:}

- fast, convenient online submission

- thorough peer review by experienced researchers in your field

- rapid publication on acceptance

- support for research data, including large and complex data types

- gold Open Access which fosters wider collaboration and increased citations

- maximum visibility for your research: over $100 \mathrm{M}$ website views per year

At BMC, research is always in progress.

Learn more biomedcentral.com/submissions 